\title{
Multiobjective Optimal Reactive Power Flow Using Elitist Nondominated Sorting Genetic Algorithm: Comparison and Improvement
}

\author{
Zhihuan $\mathrm{Li}^{\dagger}$, Yinhong $\mathrm{Li}^{*}$ and Xianzhong Duan*
}

\begin{abstract}
Elitist nondominated sorting genetic algorithm (NSGA-II) is adopted and improved for multiobjective optimal reactive power flow (ORPF) problem. Multiobjective ORPF, formulated as a multiobjective mixed integer nonlinear optimization problem, minimizes real power loss and improves voltage profile of power grid by determining reactive power control variables. NSGA-II-based ORPF is tested on standard IEEE 30-bus test system and compared with four other state-of-the-art multiobjective evolutionary algorithms (MOEAs). Pareto front and outer solutions achieved by the five MOEAs are analyzed and compared. NSGA-II obtains the best control strategy for ORPF, but it suffers from the lower convergence speed at the early stage of the optimization. Several problem-specific local search strategies (LSSs) are incorporated into NSGA-II to promote algorithm's exploiting capability and then to speed up its convergence. This enhanced version of NSGA-II (ENSGA) is examined on IEEE 30 system. Experimental results show that the use of LSSs clearly improved the performance of NSGA-II. ENSGA shows the best search efficiency and is proved to be one of the efficient potential candidates in solving reactive power optimization in the real-time operation systems.
\end{abstract}

Keywords: Optimal reactive power flow, Elitist nondominated sorting genetic algorithm, Pareto optimal, Multiobjective evolutionary algorithms

\section{Introduction}

Optimal reactive power flow (ORPF) is to optimize the steady state performance of a power system in terms of one or more objective functions, by optimally setting the control variables such as tap ratio of transformers, output of shunt compensators, and reactive power output of generators, while maintaining voltages of load buses and reactive power outputs of generators within limits. ORPF is one of the most cost-effective measures to improve voltage level, decrease network losses and maintain the power system running under normal conditions [1].

Traditionally, the objective of ORPF is to minimize real power loss of the network to obtain economic benefits [2]. To promote economic and secure operations of power systems, ORPF is developed into a multiobjective problem that involves objectives such as economical operating condition of the system, system security margin, and voltage deviation of the system, etc [3]. Usually, multiple objectives are converted to a single objective function based on preference parameters predefined by system operators. Weighted-sum method [4] allocates a weighting factor for each objective and use a linear combination as the new objective. Fuzzy-based methods draw out a membership function for each objective function to describe its satisfy-

$\dagger \quad$ Corresponding Author: Dept. of Electrical and Electronic Engineering, Huazhong Univ. of Sci. and Tech., China (zhihuan.lee@gmail.com)

* Dept. of Electrical and Electronic Engineering, Huazhong Univ. of Sci. and Tech., China

Received : December 11, 2009; Accepted : February 8, 2010 ing level [5]. In $\varepsilon$-constraint method, one of multiple objectives is chosen as the primary objective and the others are converted into inequality constraints with tradeoff tolerance parameters [6]. Goal-attainment method determines desired goal for each objective as well as a vector representing the preference direction [7]. In spite of the wide application of these objective-converting approaches in power systems, they share the characteristic that preference parameters are difficult to determine in practice. The parameter choosing and adjusting would be very timeconsuming and an inaccurate parameter configuration will lead to biasing of objective functions.

Aiming at finding a set of representative Pareto optimal solutions in a single run, multiobjective evolutionary algorithms (MOEAs) have been proposed. It is a class of stochastic optimization techniques that simulate biological evolution to solve multiobjective problems. Recent years have witnessed significant progress in the development of MOEAs. Nondominated sorting genetic algorithm (NSGA) [8], multiobjective genetic algorithm (MOGA) [9], niched Pareto genetic algorithm (NPGA) [10], strength Pareto evolutionary algorithm (SPEA) [11], elitist nondominated sorting genetic algorithm (NSGA-II) [12], etc., constitute the pioneering multiobjective approaches.

Several isolated works have demonstrated high performance of MOEAs in multiobjective ORPF [13], [14]. In spite of these applications, there is a lack of studies that compare the performance and different aspects of MOEA-based ORPF algorithms. A comparative study between NSGA-II and other four state-of-the-art MOEAs is presented for 
ORPF problem in this paper. It is demonstrated that NSGA-II obtains the best control strategy for ORPF, but it suffers from the lower convergence speed, especially at the early stage of the optimization. One of our aims is to improve the convergence speed of NSGA-II.

Generally, evolutionary techniques suffer from excessively slow convergence to locate a precise enough solution because of their failure to exploit local information [15]. Some recent work demonstrates the combination of local search strategies (LSSs) and evolutionary algorithms (EAs) can yield promising results by combining the global search ability of EAs with the local refinements advantage provided by LSSs [16]. As an approach to improve NSGAII, several problem-specific LSSs derived from previous works [17]-[19] are incorporated into NSGA-II to promote algorithm's exploiting capability and then to speed up the algorithm convergence for ORPF.

The paper is organized as follows. The ORPF is formulated as a multi-objective optimization problem in Section 2. Then, NSGA-II and four other state-of-the-art MOEAs are introduced in section 3 and their implementation for ORPF is outlined in section 4. In section 5, NSGA-II-based ORPF is tested on standard IEEE 30-bus test system and compared with four other state-of-the-art MOEAs. In section 6, several problem-specific local search strategies (LSSs) are introduced. An enhanced version of NSGA-II is proposed and examined. Section 6 concludes this paper.

\section{Problem Formulation}

\subsection{Control Variables}

ORPF is inherently a mixed-integer nonlinear programming problem involving multiple objective functions, nonlinear constraints, and both continuous variables and discrete variables. The control variables of ORPF include voltages of generators, reactive power generation of VAr sources (banks of capacitors), and tap ratios of transformers

$$
x=\left[V_{G}, Q_{c}, T_{k}\right]^{T}
$$

where $V_{G}, Q_{\mathrm{c}}$ and $T$ denotes vectors composed of generator voltages, shunt VAr compensations, and transformer tap ratios respectively, which can be written as

$$
\begin{gathered}
V_{G}=\left[V_{G, 1}, V_{G, 2} \ldots V_{G, N_{G}}\right] \\
Q_{c}=\left[Q_{c, 1}, Q_{c, 2} \ldots Q_{c, N_{c}}\right] \\
T_{k}=\left[T_{k, 1}, T_{k, 2} \ldots T_{k, N_{T}}\right]
\end{gathered}
$$

where $N_{G}, N_{c}$, and $N_{T}$ are the number of generators, the number of capacitors, and the number of shunt VAr compensations, respectively; $V_{G, i}, Q_{c, i}$ and $T_{k, i}$ are voltage magnitude at generator bus $i$, shunt reactive power at compensation bus $i$, and the tap ratio of transformer $i$.

\subsection{Minimization of Real Power Loss}

Real power loss on each individual line is calculated and the power system real power loss is calculated by summation

$$
P_{\text {loss }}(x)=\sum_{k=1}^{N_{L}} G_{k(i, j)}\left[V_{i}^{2}+V_{j}^{2}-2 V_{i} V_{j} \cos \left(\theta_{i}-\theta_{j}\right)\right]
$$

where $P_{\text {loss }}$ is real power loss; $N_{L}$ denote the number of transmission lines; $G_{k(i, j)}$ represents conductance of the $k^{\text {th }}$ line that connects bus $i$ to bus $j ; \theta_{i}$ and $\theta_{i}$ are the voltage angles at bus $i$ and bus $j$, respectively; $V_{i}$ and $V_{j}$ are the voltage magnitudes at bus $i$ and bus $j$, respectively.

\subsection{Minimization of Voltage Deviation}

To improve voltage profile of the whole system, voltage deviations from desired voltage magnitudes at load buses are summed up to calculate total voltage deviation by

$$
V_{D}(x)=\sum_{i=1}^{N}\left|\frac{V_{i}^{\text {spec }}-V_{i}}{V_{i}^{\max }-V_{i}^{\min }}\right|
$$

where $V_{D}$ is total voltage deviation; $N$ is the bus number of the power system; $V_{i}^{\text {spec }}, V_{\text {imax }}, V_{\text {imin }}$ are the desired voltage magnitude, the upper limit voltage magnitude, and the lower limit voltage magnitude at bus $i$, respectively.

\subsection{Constraints}

The above two objective functions are subject to the following operation constraints.

1) Power Balance Constraints: Power balance constraints are real and reactive power balance at each bus. They can be expressed by equality constraints

$$
\begin{aligned}
& P_{i}-V_{i} \sum_{j=1}^{N} V_{j}\left[G_{i j} \cos \left(\theta_{i}-\theta_{j}\right)+B_{i j} \sin \left(\theta_{i}-\theta_{j}\right)\right]=0, \quad 1 \leq i \leq N \\
& Q_{i}-V_{i} \sum_{j=1}^{N} V_{j}\left[G_{i j} \sin \left(\theta_{i}-\theta_{j}\right)-B_{i j} \cos \left(\theta_{i}-\theta_{j}\right)\right]=0, \quad 1 \leq i \leq N
\end{aligned}
$$

where $P_{i}$ and $Q_{i}$ are reactive and active power injections at bus $i$, and $G_{i j}$ and $B_{i j}$ denote the transfer conductance and susceptance between bus $i$ and bus $j$ respectively.

2) Control Variable Constraint: Limited to adjustment range, control variables are restricted in lower and upper boundaries

$$
\begin{array}{ll}
V_{G, i}^{\min } \leq V_{G, i} \leq V_{G, i}^{\max } & i=1 \ldots N_{G} \\
Q_{c, i}^{\min } \leq Q_{c, i} \leq Q_{c, i}^{\max } & i=1 \ldots N_{c} \\
T_{k, i}^{\min } \leq T_{k, i} \leq T_{k, i}^{\max } & i=1 \ldots N_{T}
\end{array}
$$

where $V_{G, i}^{\min }, V_{G, i}^{\max }, Q_{c, i}^{\min }, Q_{c, i}^{\max }, T_{k, i}^{\min }$, and $T_{k, i}^{\max }$ are mini- 
mum and maximum limits of the corresponding variables.

3) Dependent Variable Constraint: For secure operation, dependent variables are restricted in boundaries. These include the constraints of voltages at load buses and reactive power injection at generator buses

$$
\begin{array}{ll}
V_{i}^{\min } \leq V_{i} \leq V_{i}^{\max } & i=1 \ldots N_{P Q} \\
Q_{G, i}^{\min } \leq Q_{G, i} \leq Q_{G, i}^{\max } & i=1 \ldots N_{G}
\end{array}
$$

where $N_{P Q}$ represent the number of $P Q$ buses; $Q_{G, i}, Q_{G, i}^{\min }$, and $Q_{G, i}^{\max }$ denote reactive power generation of generator at bus $i$ and its lower and upper limits respectively.

\section{Elitist nondominated Sorting Genetic Algorithm}

Assume the objectives of a multi-objectives optimization can be expressed as

$$
\min f_{i}(x) \quad i=1,2 \ldots m
$$

where $f_{i}(x)$ denotes the $i^{\text {th }}$ objective function and $m$ is the number of objectives.

In evolutionary techniques, every individual solution should be assigned a fitness value representing its quality. This task becomes much more complex in multi-objective situations such as (14), since several objectives exist. One of the possible solutions is to divide the population into several partitions, following which the fitness assignment and selection in each partition is performed according to one of the objectives [20]. This approach possesses low efficiency, and is often shown to be sensitive to the nonconvexity of the Pareto front.

Pareto-based fitness assignment was proposed in [20] and then implemented by Deb [8] and Fonseca [9]. All these approaches explicitly use domination relations to determine the fitness of each individual solution. Based on this idea, many variations were proposed and it came out a rapidly growing interest in the area of multiobjective evolutionary algorithms (MOEAs). Please refer to [21] for indepth discussion of MOEAs.

Elitist nondominated sorting genetic algorithm (NSGAII), proposed by Deb et al. to overcome some drawbacks (high computational complexity of nondominated sorting, lack of elitism and the need for specifying a sharing parameter) of the earlier MOEAs, has been demonstrated as one of the most efficient MOEAs for benchmark problems [12].

\subsection{Pareto Concepts}

Pareto optimality is an integral part of multiobjective optimization and will be introduced first.

DEFINITION 1: A solution $x_{1}$ is said to dominate $x_{2}$ (denoted by $x_{1} \prec x_{2}$ ) if and only if:

$$
\begin{aligned}
& \forall i \in\{1,2 \ldots m\}: f_{i}\left(x_{1}\right) \leq f_{i}\left(x_{2}\right) \\
& \exists j \in\{1,2 \ldots m\}: f_{j}\left(x_{1}\right)<f_{j}\left(x_{2}\right)
\end{aligned}
$$

Usually, the Pareto concepts are defined with respect to the entire decision variable space. For MOEAs, however, these concepts are often restricted in a particular set, i.e. $S$ $=\left\{x_{i}, i=1 . . n\right\}$. Some restricted concepts and terminology are adopted.

DEFINITION 2: A solution $x$ is said to be a nondominated solution of set $S$, if $x \in S$ and there is no solution $x^{\prime} \in$ $S$ for which $x^{\prime}$ dominates $x$. A Set $P$ contains all these nondominated solutions is called nondominated set of $S$, which may be denoted by $P=\operatorname{Dom}(S)$.

DEFINITION 3: $P F=\left\{v \mid v=\left[f_{1}(x), f_{2}(x) \ldots f_{m}(x)\right]^{\mathrm{T}}, x \in\right.$ $P\}$ is said to be Pareto front of set $S$.

If replacing $S$ with populations in evolutionary techniques, e.g., population at the $k^{\text {th }}$ generation, $S^{(k)}=\left\{x_{\mathrm{i}}^{(k)}\right.$, $\left.i=1 . . n^{(k)}\right\}$, then $P^{(k)}$ and $P F^{(k)}$ can be called nondominated set and Pareto front at generation $k$, respectively.

\subsection{Fitness Assignments Schemes in NSGA-II}

Solutions dominating others are ones with superior objective values and should be given better fitness. Regarding the tasks of preventing premature convergence and providing system operator with well distributed solutions, a diverse population would be highly preferred. Therefore, Two factors are mainly considered when assigning the fitness

- The solution dominates more other solutions is prefe rred

- The solution in a sparse region is preferred.

As a first rule, NSGA-II allocates a rank value $r_{i}$ to each solution. Nondominated solutions are identified and assigned the rank value 1. After removing those solutions from the population, new nondominated solutions are assigned rank value 2 . This procedure is performed iteratively. Fig. 1 provides a graphic example. It represents the population of the $t^{\text {th }}$ generation (size 8); first, the nondominated solutions 1,2 and 3 receive rank value 1 , then solutions 4 and 5 receive rank value 2 , and the procedure continues.

To promote the solutions in the sparse region, population density estimation metric named crowding distance $D_{i}$ is

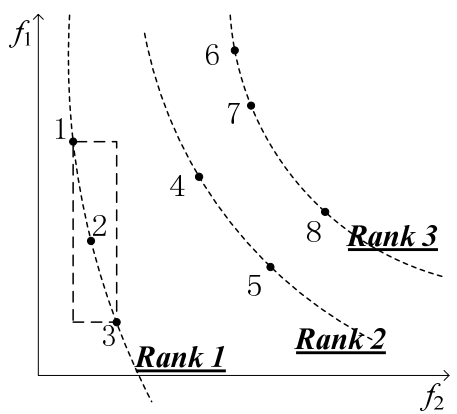

Fig.1. Fitness assignment of NSGA-II in a two-objective space. 
assigned to each candidate solution. $D_{i}$ is the average distance of two points on either side of the point $i$ along each of the objectives. For objective dimension $2, D_{i}$ for solution $i$ is determined by a rectangle formed by two nearest neighbors of $i$, as shown in Fig.1. For solution 2, solutions 1 and 3 are the two neighbors in the same rank, defining the boundary rectangle. $D_{2}$ would be the average side length of the rectangle.

After assigning $r_{i}$ and $D_{i}$, any two solutions in the population can be compared by

$$
\begin{aligned}
& \text { solution } i \text { is superior than soulution } j \Leftrightarrow \\
& \left\{r_{i}<r_{j}\right\} \text { or }\left\{r_{i}=r_{j} \text { and } D_{i}>D_{j}\right\}
\end{aligned}
$$

\subsection{MOEAs for Comparison}

Four other state-of-the-art MOEAs are introduced as comparison. To keep the integrality of this paper, they are briefly introduced.

1) Multi-objective Genetic Algorithm (MOGA): Multiobjective Genetic Algorithm (MOGA) was proposed by Fonsca and Fleming [9]. A ranking scheme is proposed in which the rank value of a certain individual corresponds to one plus the number of other individuals in the current population by which it is dominated. Then fitness sharing based on niche information is performed to distribute the population uniformly along the Paretooptimal region.

2) Nondominated Sorting Genetic Algorithm (NSGA): Nondominated Sorting Genetic Algorithm was developed by Srinivas and Deb in [8]. Analogously to MOGA, at first, the nondominated solutions in the population are identified and are assigned a same dummy fitness value. Then the second level of nondominated solutions are identified, a dummy fitness value, which is a little smaller than the worst shared fitness value before is assigned. This process is continued until all population members are assigned a shared fitness value.

3) Strength Pareto Evolutionary Algorithm (SPEA): Zitzler and Thiele presented SPEA as a potential algorithm for multi-objective optimization [11]. They suggested maintaining an external population at every generation storing all nondominated solutions discovered so far. SPEA assigns a real value called strength for each individual in the Pareto-optimal set. Subsequently, the fitness of each individual in the population is the sum of the strengths of all external Pareto solutions by which it is covered.

4) Improved Strength Pareto Evolutionary Algorithm (SPEA2): SPEA2 [22] is an enhanced version of SPEA. In SPEA II, instead of calculating standard Pareto rank, each individual in both main population and elitist archive is assigned a strength value, which incorporates both dominated and density information. On the basis of the strength value, the final rank value is determined by the summation of the strengths of the individuals that dominate the current one. Meanwhile, the nearest neighbor density estimation method is applied to obtain the density value of each individual.

\subsection{Evolving and Filtering}

NSGA-II and the four other MOEAs can be classified into two groups. SPEA, SPEA2 and NSGA-II are elitist algorithms with a filtering process that carries competitive solutions directly to the next population. MOGA and NSGA are non-elitist algorithms.

The procedures at generation $k$ are demonstrated in Fig.2. $S^{(k)}$ and $T^{(k)}$ are main population and external population at generation $k$ respectively. For MOGA and NSGA, $S^{(k)}$ only go through evolving process (reproduction, crossover, and mutation) and then $S^{(k+1)}$ is produced.

Two populations are maintained in SPEA and SPEA2. For SPEA, $S^{(k)}$ and $T^{(k)}$ would be combined and enter evolving process to obtain $S^{(k+1)}$, from which $T^{(k+1)}$ will be selected and then enter next generation together with $S^{(k+1)}$. For SPEA2, $T^{(k)}$ will go through evolving process alone to get $S^{(k+1)} . T^{(k)}$ and $S^{(k+1)}$ will be filtered and $T^{(k+1)}$ is generated.

In NSGA-II, evolving process generates the temporary new population $S_{1}{ }^{(k+1)}$ from $S^{(k)}$. Next, filtering process combines $S^{(k)}$ and $S^{(k)}$, then chooses superior solutions based on (15) to form the new population $S^{(k+1)}$.

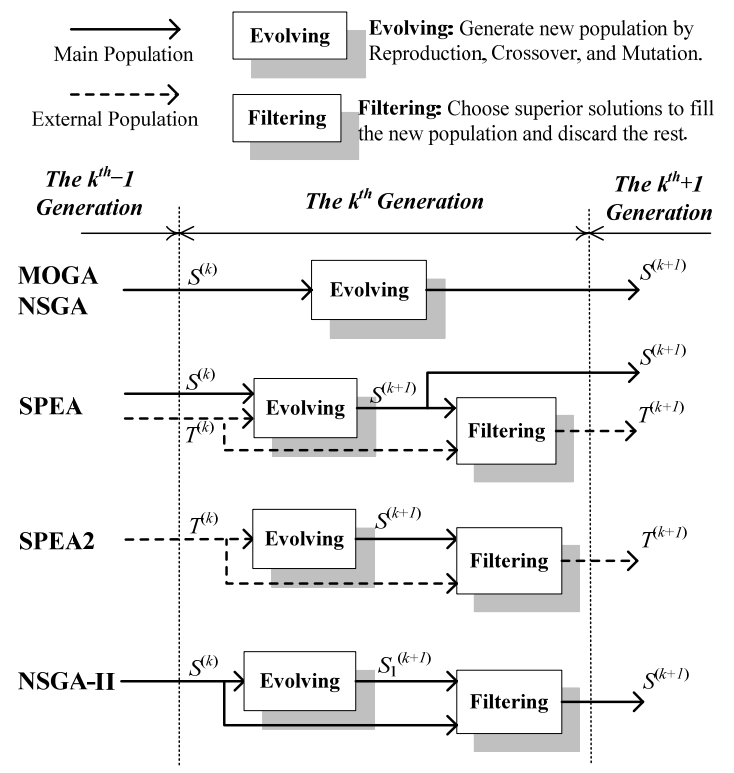

Fig.2. MOEA Procedure at generation $t$.

\section{The Implementation of MOEAs for ORPF}

\subsection{Real Coded Scheme and Constraint-handling Approach}

Five different MOEAs for ORPF are to be analyzed and compared. For reasons of fairness, the following modifications have been incorporated in all MOEAs.

1) Coding: Real-coding scheme is adopted in all MOEAs because of difficulties of binary representation when dealing with continuous search space with large dimen- 
sions. A decision variable is represented by a real number within its lower limit and upper limit. Since parts of decision variables are discrete, a procedure is imposed to round discrete variables of newly generated solutions to the nearest valid value.

2) Crossover and Mutation: A Blend Crossover- $\alpha$ operator Normally Distributed Mutation operator [21] is employed for real-coding scheme.

4) Constraint-handling approach: The overall constraints violations can be computed as

$$
F_{v}=w_{v} \sum_{i=1}^{N_{P Q}} \frac{\left|V_{i}-V_{i}^{\lim }\right|}{V_{i}^{\max }-V_{i}^{\min }}+w_{Q} \sum_{i=1}^{N_{G}} \frac{\left|Q_{G, i}-Q_{G, i}^{\lim }\right|}{Q_{G, i}^{\max }-Q_{G, i}^{\min }}
$$

where $V_{i}^{\text {lim }}$ and $Q_{G i}^{\text {lim }}$ denote the violated upper or lower limits; $w_{V}$ and $w_{O}$ are weighting coefficients that are set to 0.5 . The constraint-handling approach in the original NSGA-II is adopted. We simply incorporate constrainthandling into the definition of domination. Regarding two solutions, when both of them are feasible, the traditional definition of domination (Definition 1) still work. Otherwise, we can define that a solution with a smaller overall constraint violation (may be zero) dominate the other solution. Using this definition, most of MOEAs can be easily extended to solve ORPF with constraints and the search will be guided towards feasible region as well as the nondominated set.

\subsection{The Flow Chart of the Algorithms}

The flow chart is shown in Fig.2.

1) System Initialization: Input the data of electric power systems including branches, buses and parameters of devices such as generator voltages range, transformer tap ratios, and the range of shunt capacitors. Calculate admittance matrix;

2) Algorithm Initialization: Randomly generate initial population; set $k=1$; configure algorithm parameters;

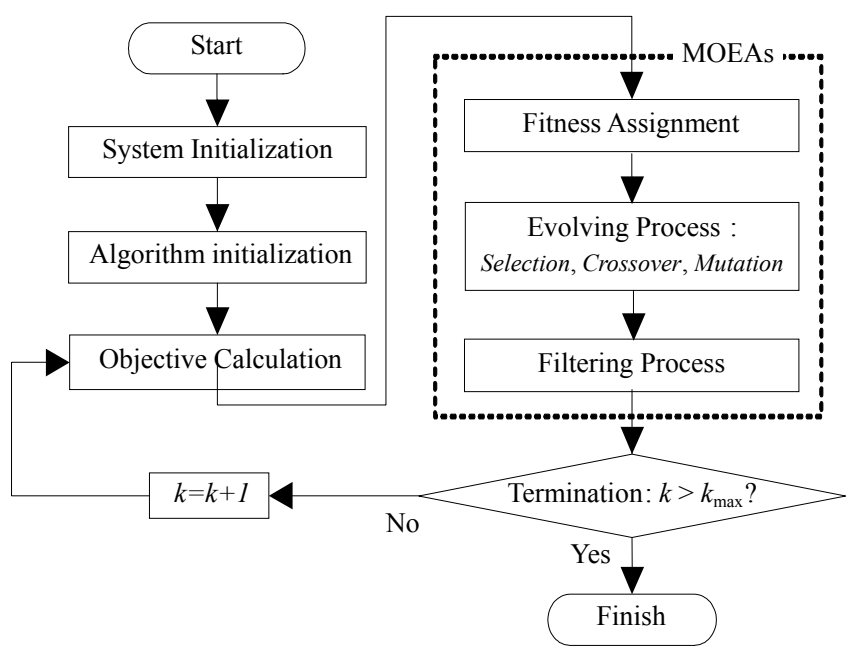

Fig.3. Flow chart of MOEAs for ORPF.
3) Objective Calculation: For each individual, a load flow calculation for (7) and (8) is performed, and real power loss as well as voltage deviation are calculated by (5) and (6). When any inequality constraint is violated, the overall violations of constraints should be computed by (16).

4) MOEAs Part: As demonstrated in Fig.3, fitness assignment will be performed first as mentioned in section 3 . Then evolving process and filtering process can be invoked as in Fig.2.

5) Termination: Check whether the maximum iteration quantity is reached. If not, $k=k+1$ and go to the step (3).

\section{Case Study}

\subsection{System Data}

IEEE 30-bus system is used as the test system. The network data and initial operating conditions are given in [23]. The network consists of 41 branches, 6 generator-buses, and 22 load-buses. Shunt compensators are installed at bus 10 and bus 24. Control variables include 6 generator voltages, 4 transformer tap ratios, and 2 shunt capacitors. Settings of the decision variables are listed in Table 1, where $Q_{C, 10}$ and $Q_{C, 24}$ represent shunt compensators at bus 10 and bus 24 respectively. All the parameters are in the per unit system. The upper and lower limits of the generator voltages are set to 1.1 p.u. and 0.9 p.u. respectively.

Table 1. Decision variable settings

\begin{tabular}{c|c|c|c}
\hline-- & Upper limits & Lower limits & Tap \\
\hline$V_{G}$ & 1.1 & 0.9 & Continues \\
\hline$T_{k}$ & 1.1 & 0.9 & $0.0125 \times 16$ \\
\hline$Q_{C, 10}$ & 0.19 & 0 & $0.04 \times 4+0.03$ \\
\hline$Q_{C, 24}$ & 0.04 & 0 & $0.01 \times 4$ \\
\hline
\end{tabular}

\subsection{Comparison of Nondominated Solutions}

The population size and generation number are set to 100. Fig. 4 gives the plots of $P F^{(100)}$ achieved by five MOEAs. Except for NSGA, all the algorithms evolve reasonably distributed Pareto fronts and their solutions extend along distinct curves in the objective space. System operator can directly choose the optimal solution according to system states and corresponding protocols. Here, the following cases may be considered for example, and corresponding optimal solutions are compared.

Case 1: Minimizing voltage deviation and keeping the power loss below 6.9MW.

Case 2: Minimizing the power loss and keeping the voltage deviation below 4.0.

The optimal solutions in Case 1 and Case 2 for five MOEAs are marked in Fig.4, and the detailed data is listed in Table 2. ENSGA is an enhanced version of NSGA-II and will be discussed in the next section. In Case 1 when all the optimal solutions $P_{\text {loss }}$ are below $6.9 \mathrm{MW}$, the optimal solution of NSGA-II has the best voltage deviation of 4.4748, 
Table 2. Best solution in different Cases

\begin{tabular}{|c|c|c|c|c|c|c|c|c|c|c|c|c|c|c|c|}
\hline & & \multicolumn{12}{|c|}{ Control Variables } & \multicolumn{2}{|c|}{$\begin{array}{l}\text { Objective } \\
\text { Function }\end{array}$} \\
\hline & & $\begin{array}{l}U_{G 1} \\
/ \mathrm{pu}\end{array}$ & $\begin{array}{l}U_{G 2} \\
/ \mathrm{pu}\end{array}$ & $\begin{array}{l}U_{G 3} \\
/ \mathrm{pu}\end{array}$ & $\begin{array}{l}U_{G 8} \\
/ \mathrm{pu}\end{array}$ & $\begin{array}{l}U_{G 22} \\
/ \mathrm{pu}\end{array}$ & $\begin{array}{c}U_{G 13} \\
/ \mathrm{pu}\end{array}$ & $\begin{array}{l}Q_{C 10} \\
/ \mathrm{pu}\end{array}$ & $\begin{array}{l}Q_{C 24} \\
/ \mathrm{pu}\end{array}$ & $\begin{array}{l}T_{6,9} \\
/ \mathrm{pu}\end{array}$ & $\begin{array}{l}T_{6,10} \\
/ \mathrm{pu}\end{array}$ & $\begin{array}{l}T_{4,12} \\
/ \mathrm{pu}\end{array}$ & $\begin{array}{c}T_{28,27} \\
/ \mathrm{pu}\end{array}$ & $\begin{array}{c}P_{\text {loss }} \\
/ \mathrm{MW}\end{array}$ & $\begin{array}{l}V_{D} \\
/ \mathrm{pu}\end{array}$ \\
\hline \multirow[t]{4}{*}{ Case 1} & SPEA & 1.0746 & 1.0559 & 1.0226 & 1.0298 & 1.0932 & 1.0686 & 0.1900 & 0.0200 & 1.0875 & 0.9125 & 1.00 & 0.9500 & 6.8951 & 7.5984 \\
\hline & \begin{tabular}{|l|} 
SPEA2 \\
\end{tabular} & 1.0767 & 1.0630 & 1.0242 & 1.0329 & 1.0801 & 1.0671 & 0.0400 & 0.0400 & 1.0125 & 1.00 & 1.0375 & 0.9875 & 6.8987 & 4.9666 \\
\hline & NSGAII & 1.0785 & 1.0624 & 1.0262 & 1.0385 & 1.0747 & 1.0232 & 0.1900 & 0.0400 & 1.0875 & 0.9750 & 1.0125 & 0.9875 & 6.8756 & 4.4748 \\
\hline & ENSGA & 1.0739 & 1.0580 & 1.0269 & 1.0367 & 1.0327 & 1.0357 & 0.1900 & 0.0400 & 1.0750 & 0.9500 & 1.0125 & 0.9750 & 6.8950 & 4.0083 \\
\hline \multirow[t]{6}{*}{ Case 2} & MOGA & 1.0505 & 1.0361 & 1.0005 & 1.0128 & 1.0255 & 1.0756 & 0.1200 & 0.0400 & 0.9875 & 0.9375 & 1.0250 & 0.9500 & 7.1632 & 3.4522 \\
\hline & NSGA & 1.0338 & 1.0197 & 0.9937 & 1.0013 & 1.0288 & 1.0383 & 0.1900 & 0.0400 & 1.0375 & 0.9125 & 0.9875 & 0.9375 & 7.3617 & 2.0632 \\
\hline & SPEA & 1.0599 & 1.0440 & 1.0068 & 1.0224 & 1.0789 & 1.0434 & 0.1900 & 0.0200 & 1.0750 & 0.9375 & 1.0250 & 0.9500 & 7.0978 & 3.7859 \\
\hline & SPEA2 & 1.0631 & 1.0474 & 1.0156 & 1.0248 & 1.0653 & 1.0583 & 0.0400 & 0.0400 & 1.00 & 0.9875 & 1.0250 & 0.9750 & 7.0165 & 3.9229 \\
\hline & NSGAII & 1.0711 & 1.0553 & 1.0212 & 1.0312 & 1.0606 & 1.0295 & 0.1900 & 0.0400 & 1.0625 & 0.9750 & 1.00 & 0.9750 & 6.9424 & 3.9562 \\
\hline & ENSGA & 1.0733 & 1.0573 & 1.0254 & 1.0357 & 1.0345 & 1.0356 & 0.1900 & 0.0400 & 1.0750 & 0.9500 & 1.0125 & 0.9750 & 6.9038 & 3.9597 \\
\hline
\end{tabular}

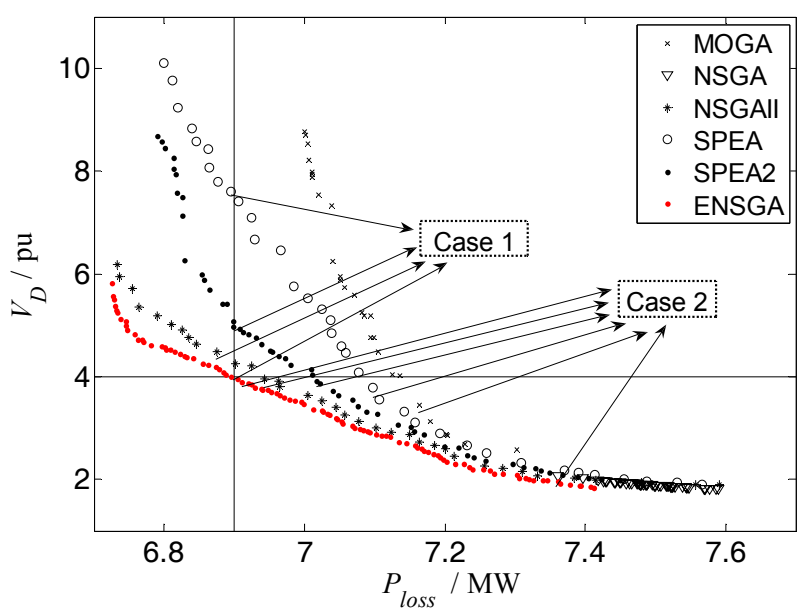

Fig. 4. Pareto fronts with MOEAs.

while SPEA2 and SPEA obtain the best voltage deviation of 4.9666 and 7.5984 respectively. No solution with power loss less than 6.9MW can be achieved by MOGA and NSGA. In Case 2 when optimal solutions have voltage deviation below 4.0, the best solution with the power loss of $6.9424 \mathrm{MW}$ is also provided by NSGA-II. The second and third best algorithms are SPEA2 and SPEA, with the power loss of 7.0165 MW and 7.0978 MW respectively. MOGA and NSGA only have the solution of 7.1632 MW and $7.3617 \mathrm{MW}$ respectively.

\subsection{Comparison of Outer Solutions}

In Fig.4, the solutions with lowest real power loss or lowest voltage deviation, which may be called outer solutions [22], locating at two edges of $P F^{(k)}$. Outer solutions may be expressed as

$$
\begin{gathered}
x_{\text {loss }}^{(k)}=\left\{x_{i} \mid \forall x_{j} \in S^{(k)}: P_{\text {loss }}\left(x_{j}\right) \leq P_{\text {loss }}\left(x_{i}\right)\right\} \\
x_{V D}^{(k)}=\left\{x_{i} \mid \forall x_{j} \in S^{(k)}: V_{D}\left(x_{j}\right) \leq V_{D}\left(x_{i}\right)\right\}
\end{gathered}
$$

where $x_{\text {loss }}^{(k)}$ and $x_{V D}^{(k)}$ are the outer solution for $P_{\text {loss }}$ and $V_{D}$ at the $k^{\text {th }}$ generation. Generally, when outer solutions with high quality are available, it is very likely that we can get a $P F^{(k)}$ with wide range, which means a better spread of solutions. In multiobjective ORPF, for system operators, outer solutions can provide the information about the extent to which each objective can be optimized. With this information, system operators can determine or modify their protocols and control strategies for operations. Since outer solutions are so important, they are compared here.

Totally 40 trials are performed. For the objective of real power loss, 40 outer solutions can be obtained at $k^{\text {th }}$ generation for each MOEA. Convergence curves that represent average value of $P_{\text {loss }}\left(x_{\text {loss }}{ }^{(k)}\right)$ are depicted in Fig.5. Fig.6 provides the information for $x_{V D}{ }^{(k)}$. The main observations from Fig. 5 and Fig. 6 can be summarized as follows.

- It is obvious that elitist MOEAs outperform the nonelitist algorithms. As demonstrated in Fig.5 and Fig.6, the curves of MOGA and NSGA converge much more slowly than the other three elitist MOEAs. This implies that the evolving of non-elitist algorithms is narrowed down in a smaller region. This observation may also be supported by Fig.4, where elitist MOEAs appears to find solutions better and widely distributed.

- Among non-elitist MOEAs, MOGA appears to be pr eferable. Regarding the objective of $P_{\text {loss }}$, a clear pe rformance gap exists between MOGA and NSGA, a nd it is also remarkable that MOGA performs well compared to SPEA in the first ten generations, altho ugh the latter one contains an elitist strategy.

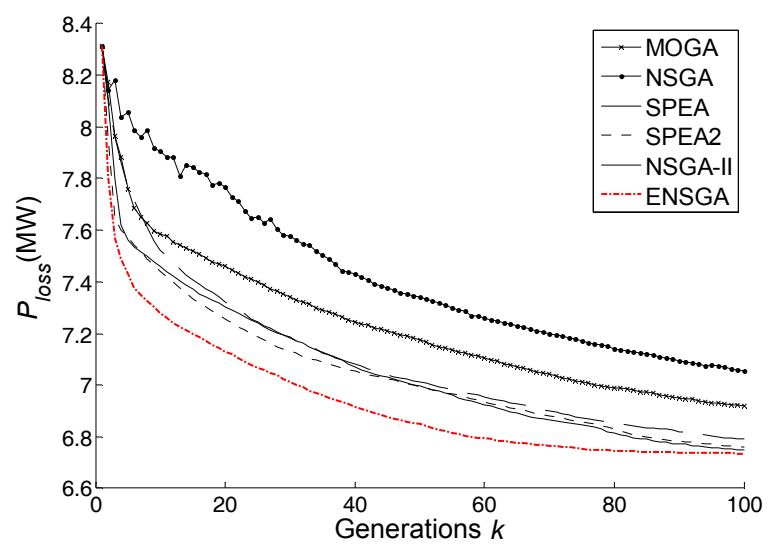

Fig. 5. Convergence of outer solutions for real power loss objective. 


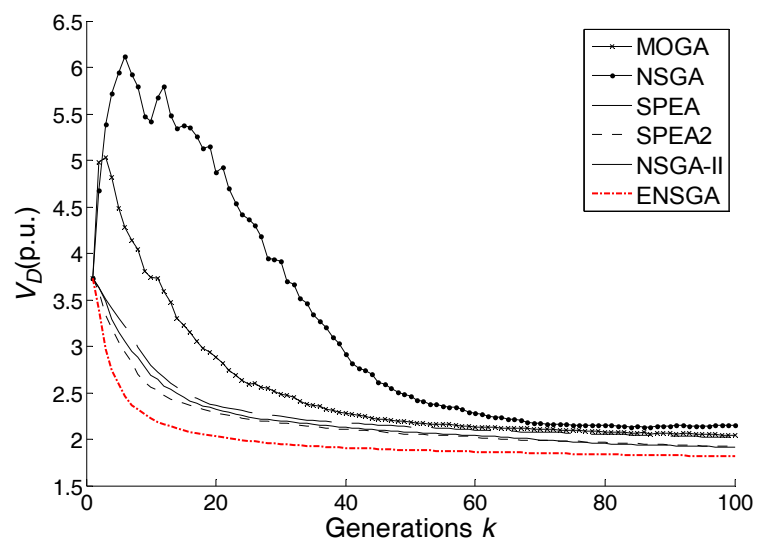

Fig.6. Convergence of outer solutions for voltage deviation objective.

- Among elitist MOEAs, the worst performance is observed with SPEA, especially for $P_{\text {loss }}$. SPEA converge much slower than SPEA2 and NSGAII in the early stage.

- The best performances are provided by NSGAII and SPEA2. Regarding real power loss objective, SPEA2 appears to do slightly better in the first 70 generations, but NSGAII catch up with SPEA2 and outperforms it after that.

By the comparison on ORPF of IEEE 30-bus system, the five algorithms can be classified into five performance lever in Table 3.

Table 3. Disadvantages of MOEAs for ORPF

\begin{tabular}{c|c}
\hline Algorithm & Performance level \\
\hline NSGA & Worst \\
\hline MOGA & Poor \\
\hline SPEA & Medium \\
\hline SPEA2 & Good \\
\hline NSGA-II & Excellent \\
\hline
\end{tabular}

\section{Improvement of NSGA-II for ORPF}

\subsection{LSSs for ORPF}

It is demonstrated that NSGA-II requires a large number of generations to converge, especially for large-scale ORPF with difficult search spaces and lengthy chromosomes. Several problem-specific local search strategies (LSSs) are introduced to increase the convergence speed.

1) Control Bus Checking LS [17]: Dependent variables at control buses are checked and corrected if corresponding constraints in (12) and (13) are violated. According to voltage's basic relation with the reactive power injection, all generators and capacitors are adjusted by (19) and (20) respectively.

$$
\begin{aligned}
& \text { if } Q_{G, i}>Q_{G, i}^{\max } \text { then } V_{G, i}=V_{G, i}-|N(0, \sigma)| \quad i=1,2 \ldots N_{G} \\
& \text { if } Q_{G, i}<Q_{G, i}^{\min } \text { then } V_{G, i}=V_{G, i}+|N(0, \sigma)|
\end{aligned}
$$

$$
\text { if } V_{c, i}<V_{c, i}^{\min } \text { then } Q_{c, i}=Q_{c, i}+\Delta Q
$$

where $N_{c}$ denote the number of capacitors; $V_{c, i}$ is voltage magnitude at capacitor bus $i ; \Delta Q$ is the reactive power change for one-tap-move of the capacitor; $N(0, \sigma)$ is a small random number in normal distribution; $\sigma$ is set to 0.01 .

2) Voltage Correction $L S$ : The bus with the most voltage violation is identified and control device near this bus is adjusted to eliminate voltage violation. The "nearest" controllers are assigned in advance using networks' topological tree-search and then nearest transformer, capacitor, or generator is used orderly to promote or decrease the voltage magnitude.

3) Controller Random LS: One controller is selected randomly and its tap position is added or dropped for one step if it's a transformer or a capacitor. For generator, one random value change is added to the generator voltage.

4) Swap Random LS [18]: Two same type controllers are chosen randomly and their control value are swapped.

5) Max-Min Random LS: One controller is selected randomly and its output is promoted or reduced to maximum or minimum.

\subsection{Enhanced NSGA-II (ENSGA) for ORPF}

Fig. 7 demonstrates the procedure of ENSGA (NSGA-II with LSSs) at $k^{\text {th }}$ generation. Compared with NSGA-II in Fig.2, after evolving process, ENSGA submits the temporary new population $S_{1}{ }^{(k+1)}$ to a local search procedure for the purpose of improving their fitness function before entering filtering process.

The main difficulty here is the comparison between a current solution and the corresponding new solution produced by LSS when they are nondominated with each other. Weighted scalar fitness function method [24] and Pareto ranking method [25] are two primary approaches to handle this situation. Some experimental results showed that better results are obtained by the weighted scalar approach than the Pareto ranking approach [26]. Weighted sum fitness function can be calculated by

$$
F_{i t}=\lambda_{1} \frac{f_{\text {loss }}-f_{\text {loss }}^{\min }}{f_{\text {loss }}^{\max }-f_{\text {loss }}^{\min }}+\lambda_{2} \frac{V_{D}-V_{D}^{\min }}{V_{D}^{\max }-V_{D}^{\min }}+\mu F_{V}
$$

where $\mu$ is the penalty coefficient for constraint violation; $f_{\text {loss }}^{\min }, f_{\text {loss }}^{\max }, V_{D}^{\min }$ are $V_{D}^{\max }$ are extreme values for two objective functions respectively, which we use the maximal and minimal objective value at each generation; $\lambda_{1}$ and $\lambda_{2}$ are non-negative weight coefficients. A set of uniformly distributed weight vectors are adopted: $(0,1),(0.2,0.8)$, $(0.4,0.6),(0.6,0.4),(0.8,0.2)$, and $(1,0)$ for $\left(\lambda_{1}, \lambda_{2}\right)$.

In a LS process in Fig. 7, 10\% percent best solutions of $S_{1}{ }^{(k+1)}$ are selected by tournament selection with replacement. Solutions are compared with each other based on 


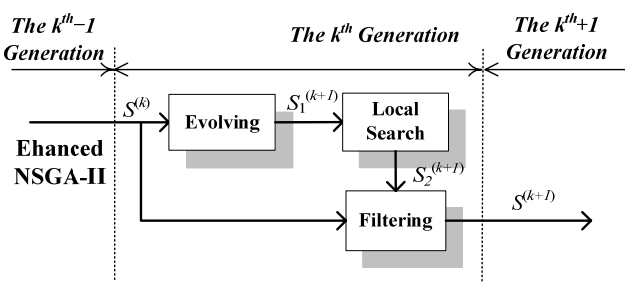

Fig. 7. The procedure of ENSGA at generation $t$.

(15). Then the problem-specific LSSs are applied sequentially to the selected solutions and several new individuals are produced. After this, a weight vector is randomly drawn from the weight vector set and is used to calculate fitness by (21) for the new individuals as well as the original solution. The one with the best fitness will be accepted. The procedure continues until all the select solutions are improved by LSSs. Next, new population $S_{1}{ }^{(k+1)}$ will enter filtering process as that in NSGA-II.

\subsection{Case Study of ENSGA}

The same procedure and genetic operators shown in Fig. 3 are used for ENSGA on multiobjective ORPF of IEEE 30-bus system. As shown in Fig.4 and Table 2, ENSGA obtains the smallest voltage deviation of 4.0083 for Case 1 and lowest system loss of 6.9038MW for Case 2.

The convergence curves of outer solutions are depicted in Fig.5 and Fig.6. Compared with NSGA-II, the convergence speed of ENSGA is increased by incorporating the problem-specific LSSs. As Fig.5 indicates, ENSGA converges before generation 60, while NSGA-II and SPEA2 locates the final solution at generation 90 and generation 100 respectively.

Table 4 and Table 5 give the best outer solution for $P_{\text {loss }}$ and $V_{D}$ at the final generation. ENSGA achieve the best assessments among the four algorithms. ENSGA gives $P_{\text {loss }}$ of $6.6846 \mathrm{MW}$, and the $V_{D}$ of 1.7308 , followed by NSGAII, which achieves $P_{\text {loss }}$ of $6.7052 \mathrm{MW}$, and $V_{D}$ of 1.7366 . SPEA2 only provide solutions with $P_{\text {loss }}$ of $6.7134 \mathrm{MW}$ and $V_{D}$ of 1.7627 , while the $P_{\text {loss }}$ and $V_{D}$ are $6.7414 \mathrm{MW}$ and 1.8214 for SPEA.

Table 4. Best Outer Solutions for Real Power Loss

\begin{tabular}{c|r|r|r|r}
\hline $\begin{array}{c}\text { Decision / } \\
\text { Objectives }\end{array}$ & SPEA & SPEA2 & NSGAII & ENSGA \\
\hline$V_{G 1}$ & 1.0843 & 1.0827 & 1.0808 & 1.0858 \\
\hline$V_{G 2}$ & 1.0666 & 1.0637 & 1.0673 & 1.0688 \\
\hline$V_{G 5}$ & 1.0389 & 1.033 & 1.0447 & 1.0389 \\
\hline$V_{G 8}$ & 1.0468 & 1.0497 & 1.052 & 1.0511 \\
\hline$V_{G 11}$ & 1.0516 & 1.0899 & 1.0652 & 1.0856 \\
\hline$V_{G 13}$ & 1.0585 & 1.084 & 1.0891 & 1.0726 \\
\hline$Q_{C 10}$ & 0.16 & 0.16 & 0.16 & 0.16 \\
\hline$C_{C 24}$ & 0.04 & 0.04 & 0.03 & 0.03 \\
\hline$T_{6,9}$ & 1.0375 & 1.025 & 1.0000 & 1.0125 \\
\hline$T_{6,10}$ & 0.9750 & 0.95 & 0.9875 & 0.9875 \\
\hline$T_{4,12}$ & 1.0375 & 1.0125 & 1.0000 & 0.9875 \\
\hline$T_{28,27}$ & 0.9875 & 0.975 & 0.9625 & 0.9625 \\
\hline$P_{\text {loss }}(\mathrm{MW})$ & 6.7414 & 6.7134 & 6.7052 & 6.6846 \\
\hline & & & & \\
\hline
\end{tabular}

Table 5. Best Outer Solutions for Voltage Deviation

\begin{tabular}{c|c|c|c|c}
\hline $\begin{array}{c}\text { Decision / } \\
\text { Objectives }\end{array}$ & SPEA & SPEA2 & NSGAII & ENSGA \\
\hline$V_{G 1}$ & 1.0300 & 1.0244 & 1.0277 & 1.0289 \\
\hline$V_{G 2}$ & 1.0159 & 1.0118 & 1.0119 & 1.0177 \\
\hline$V_{G 5}$ & 1.0014 & 1.0048 & 1.0022 & 1.0044 \\
\hline$V_{G 8}$ & 1.0016 & 1.0059 & 1.0011 & 1.006 \\
\hline$V_{G 11}$ & 1.0060 & 1.0043 & 1.0009 & 1.0043 \\
\hline$V_{G 13}$ & 1.0317 & 1.037 & 1.0248 & 1.0217 \\
\hline$Q_{C 10}$ & 0.1900 & 0.19 & 0.19 & 0.19 \\
\hline$C_{C 24}$ & 0.0400 & 0.04 & 0.04 & 0.04 \\
\hline$T_{6,9}$ & 1.0125 & 1.0125 & 1.0125 & 1.0125 \\
\hline$T_{6,10}$ & 0.9250 & 0.925 & 0.9 & 0.9125 \\
\hline$T_{4,12}$ & 0.9625 & 0.975 & 0.9625 & 0.9625 \\
\hline$T_{28,27}$ & 0.9375 & 0.9375 & 0.9375 & 0.9375 \\
\hline$V_{D}$ (p.u.) & 1.8214 & 1.7627 & 1.7366 & 1.7308 \\
\hline
\end{tabular}

\section{Conclusion}

Elitist nondominated sorting genetic algorithm (NSGAII) is applied to optimal reactive power flow (ORPF) problem in this paper and an enhanced version of NSGA-II (ENSGA) with several problem-specific local search strategies (LSSs) is proposed. A comparative study between NSGA-II and other four state-of-the-art multiobjective evolutionary algorithm (MOEAs) is also carried out for ORPF problem. The five state-of-the-art MOEAs and ENSGA have been evaluated on IEEE 30 bus systems and the Pareto fronts and outer solutions were compared. Experimental results validated the effectiveness of NSGA-II and also demonstrated the further performance improvement in ENSGA brought by the incorporation of LSSs. ENSGA was found to be one of the efficient potential candidates in solving reactive power optimization. Its application on an energy control centre of large-scale power systems should be investigated for future works.

\section{Acknowledgements}

This work was supported by National Natural Science Foundation of China (NSFC) (No. 50595414), by the National Basic Research Program of China ("973" Projects) (2004CB217906), and by National Key Technology R\&D Program (No. 2008BAA13B00).

\section{References}

[1] Hwa-Chang Song, Byong-Jun Lee, and Young-Hwan Moon, "An Interior Point Method based Reactive Optimal Power Flow Incorporating Margin Enhancement Constraints," KIEE International Transactions on Power Engineering, Vol.5-A, No.2, pp.152-158, 2005.

[2] D. Pudjianto, S. Ahmed, and G. Strbac, "Allocation of VAR supportusing LP and NLP based optimal power flows," IEE Proc. Generation, Transmission, and 
Distribution, Vol.149, No.4, pp.377-383, 2002.

[3] R. He, G. A. Taylor, and Y. H. Song, "Multi-objective optimal reactive power flow including voltage security and demand profile classification," International Journal of Electrical Power \& Energy Systems, Vol. 30, pp.327-336, 2008.

[4] Y.-j. Zhang and Z. Ren, "Real-time optimal reactive power dispatch using multi-agent technique," Electric Power Systems Research, Vol.69, pp.259-265, 2004.

[5] B. Venkatesh and R. Ranjan, "Fuzzy EP algorithm and dynamic data structure for optimal capacitor allocation in radial distribution systems," Generation, Transmission and Distribution, IEE Proceedings-, Vol. 153, pp.80-88, 2006.

[6] C. Yuan-Lin, "Weak bus-oriented optimal multi-objective VAr planning," Power Systems, IEEE Transactions on, Vol.11, pp.1885-1890, 1996.

[7] C. Yuan-Lin and L. Chun-Chang, "Optimal multiobjective VAr planning using an interactive satisfying method," Power Systems, IEEE Transactions on, Vol. 10, pp.664-670, 1995.

[8] N. Srinivas and K. Deb, "Multiobjective optimization using nondominated sorting in genetic algorithms," Evol. Comp. J., Vol.2, pp.221-248,1995.

[9] C. M. Fonseca and P. J. Fleming., "Multiobjective optimization and multiple constraint handling with evolutionary algorithms-part I: a unified formulation," System, Man, Cybernetics, IEEE Transaction on, Vol.3, pp.26-37, 1998.

[10] Horn, J. Nafpliotis, N. Goldberg, D. E. "A niched Pareto genetic algorithm for multiobjective optimization Evolutionary Computation," in Proceedings of the First IEEE World Congress on Computational Intelligence. 1994,

[11] E. Zitzler and L. Thiele, "Multiobjective evolutionary algorithms: a comparative case study and the strength Pareto approach," Evolutionary Computation, IEEE Transactions on, Vol.3, pp.257-271, 1999.

[12] K. Deb, A. Pratap, S. Agarwal, and T. Meyarivan, “A fast and elitist multiobjective genetic algorithm: NSGA-II," Evolutionary Computation, IEEE Transactions on, Vol.6, pp.182-197, 2002.

[13] Abido M A, Bakhashwain J M "Optimal VAR dispatch using a multiobjective evolutionary algorithm," International Journal of Electrical Power \& Energy Systems. Vol.27, pp.13-20, 2005.

[14] Varadarajan M and Swarup K S. "Solving multiobjective optimal power flow using differential evolution," IET Generation, Transmission \& Distribution. Vol.2, No.5, pp.720-730, 2008.

[15] N. Krasnogor and J. Smith, "A tutorial for competent memetic algorithms: Model, taxonomy and design issues," IEEE Trans. Evol. Comput., Vol.9, No.5, pp. 474-488, Oct., 2005.

[16] W. Hart, N. Krasnogor, J. Smith, Eds. Recent Advances in Memetic Algorithms. Berlin, Germany: Springer-Verlag, 2004.

[17] Iba K. "Reactive power optimization by genetic algorithm," IEEE Transactions on Power Systems. Vol.9, No.2, pp.685-692, 1994.
[18] Bhagwan D D and Patvardhan C. "A new hybrid evolutionary strategy for reactive power dispatch," Electric Power Systems Research. Vol.65, No.2, pp.83-90, 2003.

[19] Bakirtzis A G Biskas P N Zoumas C E, et al. "Optimal power flow by enhanced genetic algorithm," IEEE Transactions on Power Systems. Vol.17, No.2, pp.229-236, 1994.

[20] Goldberg D E Genetic. Algorithms in Search, Optimization, and Machine Learning. Reading. Reading, Addison-Wesley, 1989

[21] A. Abraham, L. Jain, R. Goldberg. Evolutionary multiobjective optimization: theoretical advances and applications. New York: Springer Science, 2005

[22] E. Zitzler, M. Laumanns, and L. Thiele. SPEA2: Improving the Strength Pareto Evolutionary Algorithm. Swiss Federal Institute of Technology, Lausanne, Switzerlnd, Tech. Rep. TIK-Rep. 103, 2001.

[23] Z. Boming, C. Shousun, High Electric Power Network Analysis, Publisher of Tsinghua University, Beijing, 1996, pp.311-313.

[24] Jaszkiewicz A (2004) On the computational efficiency of multiple objective metaheuristics: the knapsack problem case study. Eur J Oper Res 158:418-433

[25] Murata T, Kaige S, and Ishibuchi H. "Generalization of dominance relation-based replacement rules for memetic EMO algorithms," Lect Notes Comput Sci 2723, pp: 1233-1244, 2003

[26] Hisao Ishibuchi and Kaname Narukawa. Some Issues on the Implementation of Local Search in Evolutionary Multiobjective Optimization. GECCO 2004, LNCS 3102, pp.1246-1258, 2004.

Zhihuan Li He received the B.Eng. degree in 2005, and M.S degree in 2007, from Huazhong University of Science and Technology, Wuhan, China, where he is currently pursuing the $\mathrm{Ph} . \mathrm{D}$. degree.His research interests include power system reactive power optimization and control, computational intelligence applications, and parallel computing.

Yinhong Li She received her B.S and Ph.D. degrees in Electrical Engineering from Huazhong University of Science and Technology, Wuhan, China in 1998 and 2004 respectively. Dr. Li is an associate professor in the College of Electrical and Electronic Engineering at HUST. Her current research includes relay coordination, voltage stability, and load modeling.

Duan Xianzhong He received the B.Eng. degree and the Ph.D. degree in electrical engineering from Huazhong University of Science and Technology, Wuhan, China, in 1987 and 1992, respectively. Currently, he is a professor in the Department of Electrical Engineering, and also the vice precident of Huazhong University of Science and Technology. His research interests include power system analysis and planning, voltage stability, flexible ac transmission system, and application of information technology in the power system. 